\title{
Mechanistic Insights of Empagliflozin-Mediated Cardiac Benefits: Nearing the Starting Line
}

\author{
Editorial to: "Empagliflozin Improves Left Ventricular Diastolic Dysfunction in a Genetic \\ Model of Type 2 Diabetes" by N. Hammoudi et al.
}

\author{
Manisha Gupte ${ }^{1} \cdot$ Prachi Umbarkar $^{1} \cdot$ Hind Lal $^{1}$ (D)
}

Published online: 21 July 2017

(C) Springer Science+Business Media, LLC 2017

Type 2 diabetes mellitus (T2DM), which affects 29 million Americans, is a major risk factor for the development of cardiovascular diseases (CVD) such as myocardial infarction and heart failure (HF). In the last decade, empagliflozin, a sodium glucose co-transporter 2 (SGLT-2) inhibitor, has emerged as a novel and promising drug for T2DM patients with benefits extending beyond its glucose-lowering action [1]. These comprise weight loss, blood pressure reduction, lessening arterial stiffness, and a remarkable drop in cardiovascular death [2-5]. This drug elicits its glucose-lowering effect by inhibiting SGLT-2, thereby decreasing glucose reabsorption in the proximal tubule and increasing glucose excretion in the urine [6]. While the glucose-lowering effect of empagliflozin is well recognized, the cardiovascular benefits were unexpected. The first study that reported the protective role of empagliflozin on cardiovascular outcomes was the EMPAREG OUTCOME trial, which included 7020 T2DM patients from 42 countries with a high risk of cardiovascular events [3]. Enrolled subjects received either empagliflozin once daily (10 or $25 \mathrm{mg}$ ) or placebo in addition to standard care. Surprisingly, a significant reduction in death from cardiovascular cause, hospitalization from heart failure, and percent of death from any cause were noticed in the case of patients receiving empagliflozin. Subsequently, several other clinical trials confirmed the cardiac benefits of empagliflozin in patients with or without baseline heart failure [4, 5]. Kaku et al. [5] investigated the potential cardiovascular benefits of empagliflozin treatment in the Asian population. Indeed,

Hind Lal

hind.lal@vanderbilt.edu

1 Division of Cardiovascular Medicine, Vanderbilt University Medical Center, 2220 Pierce Ave., Suite PRB\#348A, Nashville, TN 37232, USA
T2DM patients receiving empagliflozin showed significantly reduced cardiovascular associated events. While the available data provide a strong evidence of empagliflozin benefits in reducing cardiac mortality and morbidity, the mechanism through which empagliflozin exerts its cardiac benefits is unknown. Therefore, an important challenge remaining is to elucidate the molecular mechanism of empagliflozin-mediated cardiac benefits.

The study by Hammoudi et al. [7] in this issue of Cardiovascular Drugs and Therapy examined the underlying signaling mechanism of empagliflozin-mediated cardiac benefits in a mouse model of T2DM. Hammoudi et al. employed a comparatively simple study design with three experimental groups: the first group included lean mice ob/ob which served as controls, the second group included ob/ob mice that were fed a diet containing vehicle, and the third group included ob/ ob mice fed with a diet mixed with empagliflozin $(10 \mathrm{mg} / \mathrm{kg} /$ day) for 6 weeks. Empagliflozin treatment significantly lowered $\mathrm{HbA1c}$, improved diastolic function (reduced E velocity and deceleration time), and increased left ventricle compliance (increased end diastolic pressure-volume relationship). While there were no alterations in the systolic function at steady state, dobutamine-induced response was significantly improved with empagliflozin treatment. These observations confirm that the employed preclinical model in the present study accurately recapitulates the clinical findings with human trials. However, a particular strength of the study is the elucidation of the underlying molecular mechanisms, specifically, identification of several intrinsic pathways potentially mediating the favorable cardiac outcome (Fig. 1).

Impaired cardiac contractility and relaxation are often associated with dysregulated sarcoplasmic reticulum (SR) $\mathrm{Ca}^{2+}$ homeostasis. Therefore, Hammoudi et al. [7] tested the hypothesis that molecular machinery regulating diastolic $\mathrm{Ca}^{2+}$ levels, such as phospholamban (PLB) phosphorylation or 


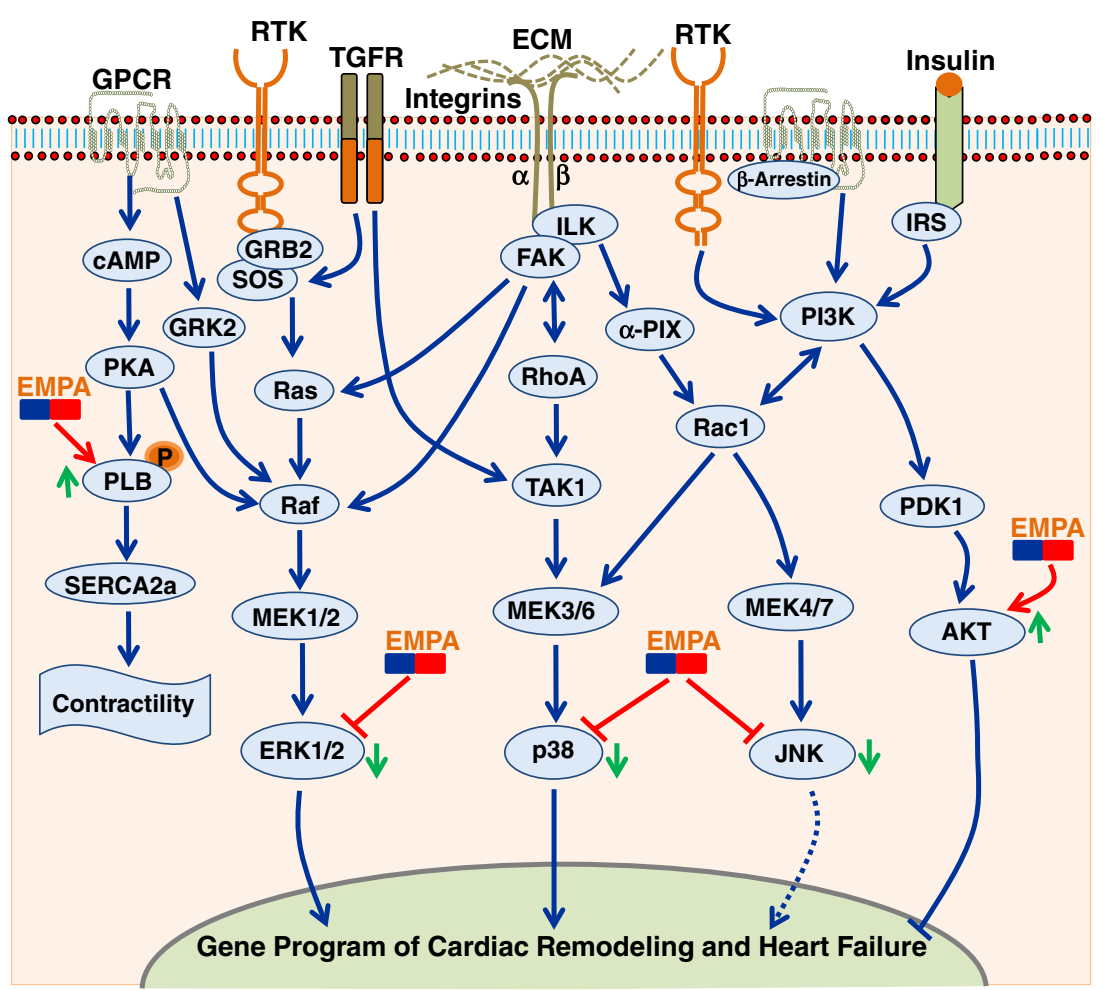

Fig. 1 Potential signaling mechanism for the beneficial effect of empagliflozin (EMPA) on cardiac function. Multiple pathways have been implicated in the myocardial pathophysiology. Many of these diverse signaling cascades culminate into MAP kinases and AKT as the final effector molecule to modulate the gene program of cardiac remodeling and heart failure. The final effectors that are modulated by EMPA are shown. AKT AKT8 virus oncogene cellular homolog, ECM extracellular matrix, $E R K$ extracellular signal-regulated kinase, $F A K$ focal adhesion kinase, GPCR G protein-coupled receptor, Grb2 growth factor receptor-bound protein $2, G R K 2 \mathrm{G}$ protein-coupled receptor kinase, ILK

SERCA2a expression, may act as downstream targets of empagliflozin. While SERCA2a expression was comparable between the groups, empagliflozin treatment led to significantly increased phosphorylation of PLB at both PLB-Ser ${ }^{16}$ and PLB-Thr ${ }^{17}$, supporting the notion that increased phospholamban activity augments the SERCA2a function leading to an improved diastolic function. However, it is not clear if empagliflozin effects on the cardiac contractility and $\mathrm{Ca}^{+}$handling are direct or a secondary outcome of overall improved metabolic effects. Further contractility experiments with isolated adult cardiomyocytes and patch clamping studies are required to address this issue. Nevertheless, considering the fact that there is no approved drug to specifically target the heart failure with preserved ejection fraction (HFpEF), the implication of these finding is enormous.

At the molecular level, empagliflozin treatment significantly blunted the activation of mitogenic stress pathways such as ERKs, JNKs, and p38 MAPKs (Fig. 1). Importantly, all these signaling cascades are strongly implicated in the pathological cardiac hypertrophy and heart failure. However, Hammoudi et al. [7] the first to identify that empagliflozin may exert integrin-linked kinase, IRS insulin receptor substrate, $J N K$ Jun N-terminal kinase, $M E K$ MAPK/Erk kinase, $P D K 1$ 3-phosphoinositide-dependent protein kinase $1, P I 3 K$ phosphoinositide 3 kinase, $P K A$ protein kinase A, $P L B$ phospholamban, Racl Ras-related $\mathrm{C} 3$ botulinum toxin substrate 1, Raf rapidly accelerated fibrosarcoma, RhoA Ras homolog gene family, member A, RTK receptor tyrosine kinase, SERCA2a sarcoplasmic/ endoplasmic reticulum $\mathrm{Ca}^{2+}$-ATPase, SOS son of sevenless guanine nucleotide exchange factor, TAK1 TGF $\beta$-activated kinase $1, T G F R$ TGF- $\beta 1$ receptor

its cardiac benefits by blunting the activation of MAPKs. As pointed out earlier, direct studies with isolated cardiomyocytes are needed to further confirm the effect of empagliflozin on the regulation of MAPK signaling in the heart. Since AKT (protein kinase B) activity plays a dynamic role in influencing both survival and ventricular contractile performance, Hammoudi et al. tested the hypothesis that cardiac AKT activity may be modulated by empagliflozin. Indeed, AKT activity was found to be preserved by empagliflozin treatment, which is known to augment the cardiomyocyte survival and contractility. Thus, Hammoudi et al were the first to identify several novel intrinsic cardiac pathways which may contribute to the observed favorable cardiac outcome of the empagliflozin treatment. Clearly, many of these findings are preliminary, proposing multiple exciting hypotheses, and needs further investigation. In agreement with the present study, numerous in vivo studies using different rodent models of diabetes have demonstrated the beneficial effect of SGLT-2 inhibition on adverse cardiac remodeling and ventricular function [8-13]. However, most of the previous studies were descriptive in nature and do not provide mechanistic insight into 
empagliflozin-mediated cardiac benefits (Table 1). Thus, this study is an important step forward in understanding the mechanism through which this class of drugs is eliciting cardiovascular benefits. However, as the authors point out, there are several limitations to the study that need to be addressed in the future work.

Multiple mechanisms have been proposed to explain the cardiac benefits of empagliflozin; these include reduced glucose, reduced plasma volume, sodium depletion, reduced blood pressure, and reduced arterial stiffness. However, all these proposed mechanisms are primarily speculation and need further clinical and experimental evidence for validation. A recent study examined the relative expression of SGLT2 and SGLT1, the two most relevant members of the SGLT family, in normal, ischemic, and hypertrophic human hearts [14]. Consistent with previous reports, Di Franco et al. [14] reported that SGLT2 does not express in either healthy or failing (ischemic/hypertrophic) human heart. However, a high-level of SGLT1 expression is seen in control hearts. Furthermore, SGLT1 expression was significantly increased in injured heart, suggesting that increased expression of
SGLT1 in cardiomyocytes may represent a potential target for empagliflozin and provides another possible explanation for the observed cardiovascular effects of empagliflozin treatment. This hypothesis can be easily tested by employing cardiac-specific SGLT1 KO animals. In order to determine whether observed benefits in the previous studies were a class effect, the CVD-REAL study compared the numbers of hospitalization due to heart failure and death in patients treated with various SGLT-2 inhibitors (dapagliflozin, canagliflozin, and empagliflozin) versus other glucose-lowering drugs (oGLDs). Consistent with the findings in the EMPA-REG OUTCOME trial, Kosiborod et al. [15] reported a reduction in hospitalization for heart failure, reduction in all-cause death, and composite reduction in hospitalization for heart failure or death, suggesting that the benefits observed in the EMPA-REG OUTCOME trial are class-related. The ongoing REFORM trial [16] will further test this hypothesis, which is a phase IV randomized, double blind trial, designed to test the efficacy of the SLGT2 inhibitor, dapagliflozin, on diabetic patients with established HF. Importantly, this critical trial will determine if SGLT2 inhibition with dapagliflozin produces

Table 1 Preclinical and clinical studies demonstrating the association of SGLT inhibition and favorable cardiac outcome

\begin{tabular}{|c|c|c|}
\hline Type of investigation & Summary of findings & Reference \\
\hline Clinical & $\begin{array}{l}\text { In the EMPA-REG trial, patients with T2D at high risk for cardiovascular events } \\
\text { received empagliflozin had significantly lower rates of the adverse cardiovascular } \\
\text { events, all-cause death, and hospitalization compared to placebo group (standard care). }\end{array}$ & [3] \\
\hline Clinical & $\begin{array}{l}\text { In a cohort of T2D with high cardiovascular risk, empagliflozin reduced heart } \\
\text { failure (HF) hospitalization and cardiovascular death in patients with and without baseline HF. }\end{array}$ & {$[4]$} \\
\hline Clinical & $\begin{array}{l}\text { In this clinical investigation, the findings of the EMPA-REG trial were further verified in } \\
\text { the Asian race. Reductions in the CV outcomes and mortality with empagliflozin were } \\
\text { consistent with the overall findings of the EMPA-REG trial. }\end{array}$ & {$[5]$} \\
\hline Preclinical (rat and rabbit) & $\begin{array}{l}\text { Effect of empagliflozin on cardiac } \mathrm{Na}^{+} / \mathrm{H}^{+} \text {exchanger (NHE) was demonstrated. } \\
\text { Specifically, empagliflozin decreases cardiac }\left[\mathrm{Na}^{+}\right]_{c} \text { and }\left[\mathrm{Ca}^{2+}\right]_{c} \text { and increases } \\
{\left[\mathrm{Ca}^{2+}\right]_{m} \text { via inhibition of the cardiac NHE. }}\end{array}$ & {$[8]$} \\
\hline $\begin{array}{l}\text { Preclinical (rats with metabolic } \\
\text { syndrome) }\end{array}$ & $\begin{array}{l}\text { Empagliflozin treatment improved cardiac inflammation, oxidative stress, } \\
\text { hypertrophy, and fibrosis. }\end{array}$ & [9] \\
\hline Preclinical (diabetic mouse) & Dapagliflozin treatment prevented the development of hypertrophic cardiomyopathy. & {$[10]$} \\
\hline Preclinical (db/db mice) & $\begin{array}{l}\text { Empagliflozin preserves the myocardial ultrastructure and improves cardiac } \\
\text { hypertrophy, fibrosis, and diastolic dysfunction. }\end{array}$ & [11] \\
\hline Preclinical (zebrafish) & $\begin{array}{l}\text { Empagliflozin treatment or knockdown of SGLT2 attenuates aristolochic acid (AA)-induced } \\
\text { heart failure in developing zebrafish embryos. }\end{array}$ & {$[12]$} \\
\hline Preclinical (ob/ob mice) & $\begin{array}{l}\text { SGLT-2 inhibition with dapagliflozin reduces the cardiac inflammation and attenuates } \\
\text { the development of diabetic cardiomyopathy in mice with T2D. }\end{array}$ & [13] \\
\hline Translational & $\begin{array}{l}\text { The relative expression of SGLT2 and SGLT1 was determined in healthy, ischemic, } \\
\text { and hypertrophic human hearts. SGLT2 does not express in the human heart either in } \\
\text { normal or pathological conditions. Whereas SGLT1 expresses in healthy heart and } \\
\text { significantly upregulated in the diseased heart. }\end{array}$ & {$[14]$} \\
\hline Clinical & $\begin{array}{l}\text { In the CVD-REAL trial, patients treated with SGLT-2 inhibitors had lower risk of heart } \\
\text { failure and death versus other glucose-lowering drugs, suggesting that the benefits } \\
\text { observed in the EMPA-REG trial may be a class effect. }\end{array}$ & {$[15]$} \\
\hline Clinical & $\begin{array}{l}\text { The ongoing REFORM trial is a phase IV randomized, double blind, placebo controlled trial, } \\
\text { designed to test the efficacy of the SLGT2 inhibitor, dapagliflozin, on diabetic patients } \\
\text { with known HF. REFORM trial identifier: https://clinicaltrials.gov/show/NCT02397421. }\end{array}$ & {$[16]$} \\
\hline Meta-analysis & $\begin{array}{l}\text { The meta-analysis data suggest that SGLT2 inhibition leads to an overall protection against } \\
\text { cardiovascular outcomes and death. }\end{array}$ & [17] \\
\hline
\end{tabular}


similar beneficial effects in patients with established HF as seen with empagliflozin in the EMPA-REG trial. Finally, a recent meta-analysis of six regulatory submissions and multiple trials with seven different SGLT-2 inhibitors confirmed the benefits of SGLT-2 inhibitors in T2DM patients against cardiovascular outcomes and mortality [17]. Additional clinical studies are urgently needed to define potential roles of empagliflozin in HFpEF patients.

In summary, this study, for the first time, provides mechanistic insight into the empagliflozin-mediated cardiac benefits; preclinical models have been optimized; signaling pathways have been identified; and only time will tell if empagliflozin is the way we should be marching for the HFpEF treatment.

\section{Compliance with Ethical Standards}

Funding This work was supported by research grants to HL from the NHLBI (R01HL133290, R01HL119234) and American Heart Association (13SDG16930103). MG was supported by Training Grant in Cardiovascular Research (T32 HL007411) from the NHLBI.

Conflict of Interest The authors declare that they have no conflict of interest.

Ethical Approval This article does not contain any studies with human participants or animals performed by any of the authors.

\section{References}

1. Abdul-Ghani MA, Norton L, DeFronzo RA. Renal sodium-glucose cotransporter inhibition in the management of type 2 diabetes mellitus. Am J Physiol Renal Physiol. 2015;309(11):F889-900.

2. Raz I, Cahn A. Heart failure: SGLT2 inhibitors and heart failureclinical implications. Nat Rev Cardiol. 2016;13(4):185-6.

3. Zinman B, Wanner C, Lachin JM, Fitchett D, Bluhmki E, Hantel S, et al. Empagliflozin, cardiovascular outcomes, and mortality in type 2 diabetes. N Engl J Med. 2015;373(22):2117-28.

4. Fitchett D, Zinman B, Wanner C, Lachin JM, Hantel S, Salsali A, et al. Heart failure outcomes with empagliflozin in patients with type 2 diabetes at high cardiovascular risk: results of the EMPAREG OUTCOME(R) trial. Eur Heart J. 2016;37(19):1526-34.

5. Kaku K, Lee J, Mattheus M, Kaspers S, George J, Woerle HJ, et al. Empagliflozin and cardiovascular outcomes in Asian patients with type 2 diabetes and established cardiovascular disease - results from EMPA-REG OUTCOME. Circ J. 2017;81:227-34.
6. Bays H. From victim to ally: the kidney as an emerging target for the treatment of diabetes mellitus. Curr Med Res Opin. 2009;25(3): 671-81.

7. Hammoudi N, Jeong D, Singh R, et al. Empagliflozin improves left ventricular diastolic dysfunction in a genetic model of Type 2 diabetes. Cardiovasc Drugs Ther 2017;31: this issue.

8. Baartscheer A, Schumacher CA, Wust RC, Fiolet JW, Stienen GJ, Coronel R, et al. Empagliflozin decreases myocardial cytoplasmic $\mathrm{Na}+$ through inhibition of the cardiac $\mathrm{Na}+\mathrm{H}+$ exchanger in rats and rabbits. Diabetologia. 2017;60(3):568-73.

9. Kusaka H, Koibuchi N, Hasegawa Y, Ogawa H, Kim-Mitsuyama S. Empagliflozin lessened cardiac injury and reduced visceral adipocyte hypertrophy in prediabetic rats with metabolic syndrome. Cardiovasc Diabetol. 2016;15(1):157.

10. Joubert M, Jagu B, Montaigne D, Marechal X, Tesse A, Ayer A, et al. The sodium-glucose cotransporter 2 inhibitor dapagliflozin prevents cardiomyopathy in a diabetic lipodystrophic mouse model. Diabetes. 2017;66(4):1030-40.

11. Habibi J, Aroor AR, Sowers JR, Jia G, Hayden MR, Garro M, et al. Sodium glucose transporter 2 (SGLT2) inhibition with empagliflozin improves cardiac diastolic function in a female rodent model of diabetes. Cardiovasc Diabetol. 2017;16(1):9.

12. Shi X, Verma S, Yun J, Brand-Arzamendi K, Singh KK, Liu X, et al. Effect of empagliflozin on cardiac biomarkers in a zebrafish model of heart failure: clues to the EMPA-REG OUTCOME trial? Mol Cell Biochem (2017).

13. Ye Y, Bajaj M, Yang HC, Perez-Polo JR, Birnbaum Y. SGLT-2 inhibition with dapagliflozin reduces the activation of the Nlrp3/ ASC inflammasome and attenuates the development of diabetic cardiomyopathy in mice with type 2 diabetes. Further augmentation of the effects with saxagliptin, a DPP4 inhibitor. Cardiovasc Drugs Ther. 2017;31(2):119-32.

14. Di Franco A, Cantini G, Tani A, Coppini R, Zecchi-Orlandini S, Raimondi L, et al. Sodium-dependent glucose transporters (SGLT) in human ischemic heart: a new potential pharmacological target. Int J Cardiol. 2017;

15. Kosiborod M, Cavender MA, Fu AZ, Wilding JP, Khunti K, Holl $\mathrm{RW}$, et al. Lower risk of heart failure and death in patients initiated on SGLT-2 inhibitors versus other glucose-lowering drugs: the CVD-REAL study. Circulation. 2017;

16. Singh JS, Fathi A, Vickneson K, Mordi I, Mohan M, Houston JG, et al. Research into the effect of SGLT2 inhibition on left ventricular remodelling in patients with heart failure and diabetes mellitus (REFORM) trial rationale and design. Cardiovasc Diabetol. 2016;15:97.

17. Wu JH, Foote C, Blomster J, Toyama T, Perkovic V, Sundstrom J, et al. Effects of sodium-glucose cotransporter-2 inhibitors on cardiovascular events, death, and major safety outcomes in adults with type 2 diabetes: a systematic review and meta-analysis. Lancet Diabetes Endocrinol. 2016;4(5):411-9. 